\title{
Technology for health: A qualitative study on barriers to using the iPad for diet change
}

\author{
Cameron Lister ${ }^{1 *}$, Joshua H. West ${ }^{1}$, Rickelle Richards ${ }^{2}$, Benjamin Crookston ${ }^{1}$, \\ Parley Cougar Hall ${ }^{1}$, Alisha H. Redelfs ${ }^{3}$

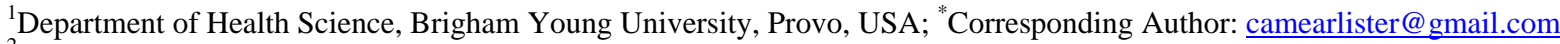 \\ ${ }^{2}$ Department of Nutrition, Dietetics, and Food Science, Brigham Young University, Provo, USA \\ ${ }^{3}$ Division of Health Promotion and Behavioral Sciences, School of Public Health, The University of Texas Health Science Center at \\ Houston, El Paso, USA
}

Received 18 February 2013; revised 22 March 2013; accepted 7 April 2013

Copyright (C 2013 Cameron Lister et al. This is an open access article distributed under the Creative Commons Attribution License, which permits unrestricted use, distribution, and reproduction in any medium, provided the original work is properly cited.

\begin{abstract}
Emergence of tablet computers has led to interest in their use to impact health-related behaviors of users. However, little is known regarding which devices are most effective for changing these behaviors, and whether or not these devices or accompanying applications (apps) are feasible or desired vehicles for behavior change. Four focus groups were conducted with students from a Western university who were given iPads to download diet-related apps. Participants were asked questions about preferences regarding iPad functionality, app functionality, and the likelihood of using apps and iPads for facilitating diet-related behavior change in the future. Two key themes emerged from focus group discussions on diet-related apps for iPads and their usefulness to facilitate behavior change. The first theme, lack of iPad practicality, contained three subthemes: inconvenient mid-way technology, internet access barriers, and smartphone preference. The second theme that emerged was attitudes towards apps in general. Three subthemes were identified under the second theme: too intensive; positive functions not specific to iPads; and lack of reliable/trustworthy information. This paper is the first, to this author's knowledge, to report the use of qualitative methods to study the practicality of using apps and iPads in changing dietrelated behaviors. Early generations of iPads may not be effective devices for facilitating behavior change among college students; and diet-related apps seem to have poor functionality that lead to cessation of use.
\end{abstract}

Keywords: iPads; Diet-Related Behavior Change; Qualitative Methods; Technology and Health; Behavior Change

\section{INTRODUCTION}

\subsection{Background}

The use of mobile devices in supporting health behavior change has been the topic of several recent research studies. Aside from expanded opportunities for users to access health information, mobile devices are becoming useful for facilitating the ongoing collection of personal data and cueing behavior change [1-3]. One of the earliest applications of using mobile devices was in 1998 when wristwatches were used to cue recovering addicts to complete logs regarding their behavior and feelings [4]. The most recent health applications have focused on a simple message system (SMS), commonly known as text messaging [5], mostly related to diabetes management [6-8] and smoking cessation [9]. Clinical care applications, or applications that assist patients manage existing conditions or illnesses, are most prevalent with fewer targeting preventive health behaviors $[6,10]$.

The recent emergence of smartphones and tablet computers, such as the iPad, has greatly enlarged both the reach and realm of possibilities for health behaviors. Worldwide, 84 million Apple iPads have been sold since its debut in 2010 [11], and 17 million alone were sold between April 2012 and June 2012 [12]. The iPad comprised 68 percent of the worldwide tablet market share during this period [11]. Additionally, 250,000 iPad applications (apps) have been downloaded [13]. The iPad is the dominant tablet computer on the market worldwide and ownership continues to increase.

The iPad provides a platform for developers to design 
third-party apps, which expand the functionality and utility of the device. The iPad's high penetration into the consumer market and the number of app downloads provide a potential method for delivering and disseminating health information. Furthermore, applications may offer an additional venue for promoting healthy behaviors through targeted health interventions. Some research has been conducted studying technology based interventions and behavior change [14]; although, most intervention research has been primarily focused on internet-based interventions [15,16].

\subsection{Previous Research}

To date very little research has been conducted on the feasibility of mobile devices in promoting behavior change. West, et al. recently conducted a content analysis of health behavior theories contained in diet-related apps [17]. This study found that overall diet-related apps score low in theoretical health behavior content, but did not address whether iPads themselves are effective facilitators of behavior change.

Despite interventions designed to change behavior, many adolescents and adults continue to consume unhealthy foods and maintain a sedentary lifestyle, which are behaviors associated with obesity $[18,19]$. More than one-third of US adults, and 17\% of youth are obese [20]. Additionally, prevention of overweight and obesity has been a topic of interest in public health policy and has become a national priority [19]. One area of influence in which mobile devices are being used is in diet-related behaviors. Pew Research Center conducted a survey in which 31 percent of app-users said they used apps to track diet [21]. The use of apps and technology provides the consumer an opportunity to be in control of their own health through tracking and monitoring their diet and physical activity.

\subsection{Research Objective}

Users' ability to examine, monitor, manage, and prevent weight-gain through the use of the iPad could produce positive health and weight outcomes. Thus, the purpose of this study was to explore the utility of iPads in facilitating diet-related behavior change. Such knowledge would advance the literature in this heretofore-neglected area of research, through use of qualitative methods to assess the utility and purpose of tablet devices for behavior change.

\section{METHODS}

\subsection{Participant Characteristics}

Four focus groups were conducted with participants (n $=20$ ) recruited from an undergraduate health class at a
Western university during September, 2010. Nineteen university students, ages $19-28$, participated in the focus groups. Participants included 3 males and 16 females, including individuals of White $(\mathrm{n}=16)$, African $(\mathrm{n}=1)$, Asian $(\mathrm{n}=1)$, and Hispanic $(\mathrm{n}=1)$ ethnic origin.

\subsection{Procedure}

The study consisted of a one-group posttest only design. Participants were given first generation iPads with the instructions that "they should use the iPad to change their diet during the next three weeks". They were given access to an account linked to the Apple App Store in order to download and use diet-related health apps. Each participant received five dollars in App Store credit and was encouraged to download both paid and free apps. The Brigham Young University Institutional Review Board approved this study and each participant provided informed consent prior to participation.

\subsection{Measurement}

After a three-week exposure to the iPad, four focus group sessions were conducted by two trained moderators in October, 2010. Focus group sessions were approximately 60 minutes each and were digitally recorded. Participants participated in only one focus group and completed a brief demographic questionnaire at the beginning of each focus group. During the focus groups, participants were asked to comment about their likes and dislikes regarding the iPad's functionality and utility for helping them to change their diet. They were also asked to discuss their future likelihood of using an iPad for diet-related behavior change (Table 1).

\subsection{Qualitative Analysis}

The focus groups were transcribed verbatim from audio recordings. The moderators analyzed the data from all 4 focus groups using a thematic analysis approach as suggested by Braun \& Clarke and Krueger \& Casey [22, 23]. Two researchers were trained as moderators in thematic analysis; they then independently coded transcripts using open-coding methods with NVivo (QSR International, v. 9), a qualitative data analysis software program. They reconciled any differences in coding and identified themes that emerged from transcript evaluation.

\section{RESULTS}

Twenty individuals were recruited into the study; however, one student (female) dropped from the study for personal reasons. The other nineteen finished the study and participated in the four focus groups.

Two key themes emerged from each of the four focus groups relating to the iPad's usefulness in facilitating 
Table 1. Focus group questions and subsequent prompts.

1. Tell me about your experience with iPads in general.

- What things do you like about it? What do you dislike?

- Is there anything you liked about them, versus what you could do on a computer?

2. Tell me about your experience with apps in general.

- What things do you like about apps? What do you dislike?

3. Types of apps for changing diet.

- What things were useful about diet apps?

- What things were not useful about diet apps?

- What made apps/iPads either easy or difficult to use?

- What made apps/iPads convenient versus inconvenient to use?

4. Feasibility of changing diet.

- What aspects about the apps helped change, or did not help change your diet?

- What aspects of iPads helped or did not help change your diet?

- Would you use an iPad to change your diet-related behaviors if it was given to you?

5. iPads compared to computers or paper for changing diet.

- How did iPads compare to using a computer? - How did iPads compare to using paper?

behavior change. The first theme, lack of iPad practicality, contained three subthemes: inconvenient mid-way technology; internet access difficulties; and smartphone preference. The second theme referred to attitudes toward apps in general. Three subthemes were identified under the second theme: positive functions not specific to iPads; intensity; and lack of reliable/trustworthy information.

\subsection{Theme 1: Lack of iPad Practicality}

The first theme that was consistently noted in the focus groups was that iPads were seen as impractical facilitators of diet-related behavior change. Many different aspects of the iPad made it difficult or inconvenient to carry and use while outside of the home. Although iPad technology was seen as appealing and entertaining to use, overall it was reviewed as a poor medium for changing this specific type of behavior by participants.

\subsubsection{Inconvenient Mid-Way Technology}

Overall, iPads were seen as "cool” but not very useful for using diet/health-related apps. The majority of participants stated that iPads were not as convenient as a smartphone, with no added advantage in usability. One user summarized that "if someone already has an iPhone and a laptop, the iPad isn't very useful, because they already have other devices". Additional commentary supported this notion: "It's like the same as a phone, just bigger, and it doesn't do as much as a laptop”. Participants repeatedly criticized the iPad for being a "mid-way technology" that didn't have all the functionality of a computer and was at times harder to use; particularly the keyboard. Moreover, participants inferred that it would be difficult to fully commit to using an iPad to manage or change their diet provided that the device is otherwise limited in comparison to a laptop computer. One participant commented that she felt "my work space as a student wouldn't really work on the iPad, and thus having to track my diet on both places... would be cumbersome".

A few of the participants expressed interest in using the iPad for diet-related behavior change. This was, however, only if the iPad were given to them freely, and not if they had to purchase one on their own. One interviewee said "if it was given to me to keep, I would definitely use it".

\subsubsection{Internet Access Difficulties}

Study participants used 1st generation iPads that did not have the option of connecting to a cellular or data network that would allow for the device to be connected continuously to the Internet without a Wi-Fi connection. Whereas the option for a data plan was available on select 1 st generation models, those used in this study were not data ready. As such, difficulties associated with Internet access were among the most commonly reported barriers to diet-related applications. For example, participants reported that it was difficult to log what they were eating for various meals when so many meals were consumed away from the home, and by implication, away from access to Wi-Fi/Internet. One participant stated, "I would say one thing was not having the Internet on it... or having problems getting the Internet in places. Which, I think I would have used it more if it had Internet on it”.

\subsubsection{Smartphone Preference}

Many participants stated that smartphones would be preferable alternatives to iPads. Compared to iPads, smartphones were seen as easier to carry around; they are an item that is rarely left behind at home. One student indicated they were, "more comfortable with [their] iPhone". In general users were more familiar with the size and usability of smartphones, and its constant use by owners made it more practical for health-related apps. "Something like counting your calories or looking up certain foods is more an iPhone type thing," another participant said, with general agreement from the rest of the group.

\subsection{Theme 2: Attitudes towards Apps in General}

The second theme that emerged from the groups was related to attitudes about the apps themselves. App technology overall seemed to be found intriguing and to some degree helpful; however, these positives were not found to be specific to iPads. Additionally, users seemed to be overwhelmed with the number of apps available 
and would often question reliability of information, and experienced frustration and usability issues.

\subsubsection{Positive Functions Not Specific to iPads}

Most of the respondents liked the ease of using apps and the functionality of a touch-screen. Participants viewed very positively the provision of information about particular foods and about eating habits. Certain app functions, for instance the ability to obtain lots of information, were well liked. One participant said, "I would say the informational aspect was nice. Just if you were between two foods (choosing), and you didn't know if one has a lot of calories...if you didn't know, then that would change my behavior in a few instances". Apps that had the function of tracking data over time so that the user could view progress were also seen positively: "I like to see my progress over time, and I think it helps you, you're like-Oh, I'm on the right track." Also, some apps had the function of raising awareness of what types of foods were consumed by the user; in particular, the unhealthy types of foods like desserts and sodium. Users liked this function; one said, "I noticed that I wasn't drinking enough water, and I drank more water since I've been using [the app]... So, that increased, and my fruit and vegetable consumption increased, just because I'd put it in.”

\subsubsection{Intensity}

Primarily, apps related to diet used calorie counters that inform users whether or not their eating behaviors are good or bad. After the initial "wow-factor" wore off for the apps, users in general reviewed these functions as "too intensive" and "complicated". One participant explained the complexity, "with the calorie tracker things, it seemed like the [healthier]...home-cooked food you made, the harder it was [to] insert it”. The time it took to look up and enter in the serving sizes of what they ate after every meal was difficult. A participant commented that, "counting calories, it's really hard [to do] if they haven't ever been taught how to do that first. So I don't know if an app can necessarily teach you, like how to implement a new habit into your life". Not all diet calorie counters featured comprehensive lists of food, so finding what was eaten was difficult for many participants. Similarly, if a user skipped entering a meal or there wasn't internet access to enter a meal, recalling what they ate previously was a challenge.

Conversely, apps that were not intensive enough were seen as "useless" or "unhelpful". Generally, these comments were about free apps, while problems with the amount of effort and time that was needed to use the app referred to purchased apps. Others questioned whether or not they would use apps at all for diet, a student saying “if I'm going to like work on my health, I don't think I'd use the apps or that iPad for it”. This speaks to the possibility that in changing behavior, some people may prefer a more rudimentary approach and not intensive technological devices.

\subsubsection{Lack of Reliable/Trustworthy Information}

Participants reported difficultly in selecting apps that they thought would be useful, often deferring to the App Store's rating system. Many questioned the validity of the information provided, stating that they worried about "commercial bias" getting in the way of providing reliable information. A participant who expressed this concern described an app where "the whole thing is just trying to slang [trying to sell/advertise] their cereal bars and stuff". Furthermore, participants questioned the accuracy of some of the calorie tracking apps. Indeed, they reported taking a lot of time to enter in the food that they had eaten and got back what seemed like inaccurate reports, especially when entering in home-cooked meals. One participant stated that "everybody's foods are different, because the brand names aren't all the same... Like they're different calories for every type, so I don't feel like it's very accurate". This lack of perceived reliable information diminished participants' interest in using diet-related apps on the iPad in the future.

In response to participants' concerns about quality and reliability, some recommended that there should be a better system for recommending apps. This recommendation was both for quality and reliability of information, as well as the functionality and usefulness of the apps. "I'd see some apps (diet apps), and they'd have [an] error in it, then I feel like I can't trust the rest of the app, because I don't know what's going to be legit," one participant summarized. Many brought up how they were reluctant to purchase apps before knowing whether or not they would be helpful and reliable. The same participant went on to state that "with paid-for apps, it's hard to...see how good the app's gonna be, because you can't...really demo it... I'd be afraid if this was like my actual money I was spending."

\section{DISCUSSION}

Themes that were developed from this study involved barriers, or impediments or obstacles to using iPads to change diet-related behaviors. Participants in this study generally reported negative perceptions toward the use of iPad and diet-related apps to address diet-related behaviors. iPads were viewed to be less convenient than smartphones and not as useful as a computer or laptop. Participants reported that although the technology and functionality of iPads has a "cool factor", over time this fades and the device becomes more cumbersome than helpful in aiding behavior change. Internet problems were also a 
significant barrier to using diet-related apps because they needed internet access to work properly. This inhibited the use of apps by participants in many cases. Of note, a recurring theme in all of the focus groups was the notion that smartphones are better alternatives to iPads for addressing diet-related behaviors, even though this was not an initial topic of the study. Specifically, these findings related to both aims of this paper to determine likelihood of iPad use for diet-related behavior change and overall iPad functionality.

The findings of this study fit into years of research conducted on technology-based interventions, and a recent expansion of mobile technology research. Recently, interests of researchers, companies, and software developers alike have taken to using mobile devices as means of addressing health-related behaviors [24]. Text messaging (SMS) has been incorporated into health interventions and research in clinical settings [25] and with smoking cessation [26]. Mobile technology has been applied to and studied with ecological momentary interventions (EMIs) and has been proven effective in addressing various health behaviors [27] like smoking cessation [28], alcohol treatment [29], and physical activity [30]. Smartphones are quickly becoming the most prevalent phone and technology used in the US [31]. Due to this widespread technology, studies have shown a significant growing impact of their use in the world of healthcare as a supplement to treatment [32], but this is not without limitations.

The majority of comments in the current study in regards to apps as aids to behavior change were negative; however, the overall usability and functionality of apps were reviewed positively. Most diet-related apps used by participants in our study either dealt with counting calories or providing ideas for recipes. Of calorie counting apps that were reviewed, they were thought of as either too intensive or not intensive enough-which indicates there may be a balance that is not currently being achieved. Entering serving sizes of food consumed was also reported as being difficult and time-consuming, especially for home-cooked meals. This resulted in these types of apps being considered less usable by study participants.

Several responses in the current study supported existing views of health and technology professionals, which point towards smartphones and apps as feasible means of changing populations' behavior [31]. Specific findings in the realm of physical activity have shown promise as well as mental health. For example, participants in a 10,000 steps program were more likely to log days of walking if they had an application on a smartphone [33]. Additionally, apps have been integrated into medical treatment of mental and behavioral disorders [34]. Developers in the field of mobile health (mHealth) have begun to compile guidelines of developing healthrelated apps in order to address concerns from consumers and the professional field as well [35]. Even though the current findings indicate a lack of desire to use certain types of apps to change diet-related behavior, research in this area is still needed.

Another significant barrier identified by participants in our study was reliability of information both provided by the apps and about the apps. Commercial bias was a significant worry, as well as reliability of calorie counter results - a consequence of intensity involved in entering food information. In general, participants reported concern about not knowing which apps to purchase with no real mechanism to test reliability of information. Concern about app reliability has permeated to the medical field as doctors begin to use them more as complimentary forms of treatment [10]. This concern has led to the FDA announcing it will soon begin regulating apps designed for medical use to combat this problem [36]. A rating system developed for the app store by Apple does exist, but is based on user rating and not on reliability of information. In relation to this discovery, Happtique, a healthcare-specific Appstore, has recently announced the development of a "health app certification process" to establish which apps have credible information and utilize evidence-based approaches and practices [37]. Based on the findings of the current study, attempts at creating certification processes may work to dispel some of the concerns of users in regards to reliability of information.

Carolyn and Beth (2011) recently conducted research using focus groups to outline desired functions of physical activity applications on smartphones [38]. However, little has been researched in regards to whether or not mobile devices themselves are ideal or even desirable as mechanisms for behavior change among populations. This is one of the first studies to actually consider the users' perceptions of the technology's usability both as a whole and specifically for diet-related behaviors. However, more populations in various settings need to be studied in further detail in order to conclude whether or not mobile devices or apps can in fact help change behavior on population levels. Since mobile devices have become so prevalent and incorporated into daily life, utilizing this technology for diet and other behaviors has potential to reach and change large populations simultaneously.

\subsection{Limitations}

This study has several limitations. First the study only includes responses from college students. College students are not likely to represent technology use patterns and dietary behaviors and interests that are comparable to younger adolescents or older adults. For example, regarding dietary behaviors, college students are likely in 
pre-contemplative stages of behavior change using the Transtheoretical Model [39] rather than later stages that might be occupied by older adults. Individuals in precontemplative stages of behavior change, in relation to diet-related behaviors, would not be concerned about changing diet. In relation to iPads, these individuals would also not likely be concerned about using applications on iPads for changing their diet. Additionally, this study uses only first generation iPads with limited internet connectivity. New iterations of the iPad (e.g., iPad mini) with improved technology and functionality may provide opportunities not currently available for addressing behavior change.

Lastly, numerous responses from the focus groups concentrated on smartphones being more practical devices for using health-related apps. This was not the primary aim of the study and was not probed in the same manner that other key research questions were. Since smartphones were not the primary focus of the study, additional research should be conducted to confirm whether smartphones may be more useful facilitators of behavior change.

\subsection{Conclusion}

This paper outlines certain barriers of using mobile devices among college students and the need for developers to use best-practice methods in creating healthrelated applications. Recent research has outlined the importance of using theory-based approaches in constructing health-related apps [2,3], and consulting consumers in the development of apps to ensure that functionality matches users desires for use [38]. Since smartphones and tablets have become so common among the general population, many developers have pursued the opportunity to create apps that seek to address behavior change [31]. However, this may have been done without enough consideration of whether or not this means of behavior change is feasible, or preferred by the consumer. Aligning specifically with the aims of this study, findings suggest that iPads may not serve as the optimal facilitators of health behavior change among young college students, especially without consistent internet access.

\subsection{Recommendations}

These preliminary findings indicate the need for further research into the feasibility of using mobile devices to change behavior on population levels, including tablets. Particularly since the release of the "iPad mini", more research would need to be conducted with this new technology to see if users would prefer this smaller device for certain behaviors. Additionally, larger studies need to be conducted among different populations in the US to see if the findings of this study are in fact representative of consumer views and values.

\section{ACKNOWLEDGEMENTS}

All authors were employees or affiliates of Brigham Young University and received no grants or specific funding in order to conduct this research. The iPads were purchased by and are the property of the Health Science Department at Brigham Young University. We wish to thank James Barret and Jennifer Vehawn for moderating the focus groups, coding the transcripts into NVivo, and helping to establish the themes of this paper.

\section{REFERENCES}

[1] Patrick, K., Intille, S.S. and Zabinski, M.F. (2005) An ecological framework for cancer communication: Implications for research. Journal of Medical Internet Research, 7, e23. doi:10.2196/jmir.7.3.e23

[2] West, J.H., Hall, P.C., Hanson, C.L., Barnes, M.D., GiraudCarrier, C. and Barrett, J. (2012) There's an app for that: Content analysis of paid health and fitness apps. Journal of Medical Internet Research, 14, e72. doi:10.2196/jmir.1977

[3] Cowan, L.T., Van Wagenen, S.A., Brown, B.A., Hedin, R.J., Seino-Stephan, Y., Hall, P.C., et al. (2012) Apps of steel: Are exercise apps providing consumers with realistic expectations? A content analysis of exercise apps for presence of behavior change theory. Health Education \& Behavior, 40, 133-139. doi:10.1177/1090198112452126

[4] Litt, M.D., Cooney, N.L. and Morse, P. (1998) Ecological momentary assessment (EMA) with treated alcoholics: Methodological problems and potential solutions. Health Psychology, 17, 5. doi:10.1037/0278-6133.17.1.48

[5] Fjeldsoe, B.S., Miller, Y.D. and Marshall, A.L. (2010) MobileMums: A randomized controlled trial of an SMSbased physical activity intervention. Annals of Behavioral Medicine, 39, 101-111. doi:10.1007/s12160-010-9170-z

[6] Fjeldsoe, B.S., Marshall, A.L. and Miller, Y.D. (2009) Behavior change interventions delivered by mobile telephone short-message service. American Journal of Preventive Medicine, 36, 165-173. doi:10.1016/j.amepre.2008.09.040

[7] Katz, D.L. and Nordwall, B. (2008) Novel interactive cell-phone technology for health enhancement. Journal of Diabetes Science and Technology, 2, 147-153.

[8] Chomutare, T., Fernandez-Luque, L., Årsand, E. and Hartvigsen, G. (2011) Features of mobile diabetes applications: Review of the literature and analysis of current applications compared against evidence-based guidelines. Journal of Medical Internet Research, 13, e65. doi:10.2196/jmir.1874

[9] Obermayer, J.L., Riley, W.T. and Jersino, A.O.-M. (2004) College smoking-cessation using cell phone text messaging. Journal of American College Health, 53, 71-78. doi:10.3200/JACH.53.2.71-78

[10] Skolnik, N. and Notte, C. (2012) The quiet revolution of mobile health applications. News and Views That Matter to Psychiatrists. Clinical Psychiatry News. 
http://www.clinicalpsychiatrynews.com/views/commenta ries/single-article/the-quiet-revolution-of-mobile-health-a pplications/fbb091536048c1f718c579784baa1684.html?tx_ ttnews[sViewPointer] $=1$

[11] Sloan, P. (2012) Apple by the numbers: 84M iPads, 400M iOS devices, 350M iPods sold. News, Apple. CNET. http://news.cnet.com/8301-13579_3-57511323-37/appleby-the-numbers-84m-ipads-400m-ios-devices-350m-ipod s-sold

[12] Associated Press (2012) Number of iPads sold by Apple by quarter. MoneyWatch.

http://www.cbsnews.com/8301-505245_162-57500268/n umber-of-ipads-sold-by-apple-by-quarter

[13] Apple (2012) Apple’s app store downloads top 25 billion. Apple's Press Info.

http://www.apple.com/pr/library/2012/03/05Apples-AppStore-Downloads-Top-25-Billion.html

[14] Norman, G.J., Zabinski, M.F., Adams, M.A., Rosenberg, D.E., Yaroch, A.L. and Atienza, A.A. (2007) A review of eHealth interventions for physical activity and dietary behavior change. American Journal of Preventive Medicine, 33, 336-345. doi:10.1016/j.amepre.2007.05.007

[15] Atkinson, N.L. and Gold, R.S. (2002) The promise and challenge of eHealth interventions. American Journal of Health Behavior, 26, 494-503.

doi:10.5993/AJHB.26.6.10

[16] Neuhauser, L. and Kreps, G.L. (2010) eHealth communication and behavior change: Promise and performance. Social Semiotics, 20, 9-27. doi:10.1080/10350330903438386

[17] West, J.H., Hall, P.C., Arredondo, V., Berrett, B., Guerra, B. and Farrell, J. (2013) Health behavior theories in diet apps. Journal of Consumer Health on the Internet, 17, 115. doi:10.1080/15398285.2013.756343

[18] Menifield, C.E., Doty, N. and Fletcher, A. (2008) Obesity in America. The ABNF Journal, 19, 83-88.

[19] Velazquez, C.E., Pasch, K.E., Ranjit, N., Mirchandani, G. and Hoelscher, D.M. (2011) Are adolescents' perceptions of dietary practices associated with their dietary behaviors? Journal of the American Dietetic Association, 111, 1735-1740. doi:10.1016/j.jada.2011.08.003

[20] National health and nutrition examination survey (NHANES) (2010) National health and nutrition examination data. Centers for Disease Control and Prevention (CDC), National Center for Health Statistics (NCHS). http://www.cdc.gov/nchs/nhanes/nhanes2009-2010/nhane s09_10.htm

[21] Fox, S. and Duggan, M. (2012) Mobile health has found its market: Smartphone owners. Pew Research Center, Pew Internet and American Life Project. http://pewinternet.org/Reports/2012/Mobile-Health/KeyFindings.aspx

[22] Braun, V. and Clarke, V. (2006) Using thematic analysis in psychology. Qualitative Research in Psychology, 3, 77101. doi:10.1191/1478088706qp063oa

[23] Krueger, R. and Casey, M. (2009) Focus groups: A practical guide for applied research. 4 Edition, SAGE Publications, Thousand Oaks.
[24] Riley, W.T., Rivera, D.E., Atienza, A.A., Nilsen, W., Allison, S.M. and Mermelstein, R. (2011) Health behavior models in the age of mobile interventions: Are our theories up to the task? Translational Behavioral Medicine, 1, 53. doi:10.1007/s13142-011-0021-7

[25] Jin, W., Hollin, I. and Kachnowski, S. (2011) A review of the use of mobile phone text messaging in clinical and healthy behaviour interventions. Journal of Telemedicine \& Telecare, 17, 41-48. doi:10.1258/jtt.2010.100322

[26] Rodgers, A., Corbett, T., Bramley, D., Riddell, T., Wills, M., Lin, R.B., et al. (2005) Do u smoke after txt? Results of a randomised trial of smoking cessation using mobile phone text messaging. Tobacco Control, 14, 255-261. doi:10.1136/tc.2005.011577

[27] Heron, K.E. and Smyth, J.M. (2010) Ecological momentary interventions: Incorporating mobile technology into psychosocial and health behaviour treatments. British Journal of Health Psychology, 15, 1-39. doi:10.1348/135910709X466063

[28] Whittaker, R., Maddison, R., McRobbie, H., Bullen, C., Denny, S., Dorey, E., et al. (2008) A multimedia mobile phone-based youth smoking cessation intervention: Findings from content development and piloting studies. Journal of Medical Internet Research, 10, e49. doi:10.2196/jmir.1007

[29] Cohn, A.M., Hunter-Reel, D., Hagman, B.T. and Mitchell, J. (2011) Promoting behavior change from alcohol use through mobile technology: The future of ecological momentary assessment. Alcohol: Clinical \& Experimental Research, 35, 2209-2215. doi:10.1111/j.1530-0277.2011.01571.x

[30] King, A.C., Ahn, D.K., Oliveira, B.M., Atienza, A.A., Castro, C.M. and Gardner, C.D. (2008) Promoting physical activity through hand-held computer technology. American Journal of Preventive Medicine, 34, 138-142. doi:10.1016/j.amepre.2007.09.025

[31] Cummiskey, M. (2011) There's an app for that: Smartphone use in health and physical education. Journal of Physical Educational, Recreation \& Dance, 82, 24. doi:10.1080/07303084.2011.10598672

[32] Boulos, M.N.K., Wheeler, S., Tavares, C. and Jones, R. (2011) How smartphones are changing the face of mobile and participatory healthcare: An overview, with example from eCAALYX. Biomedical Engineering Online, 10, 24 37. doi:10.1186/1475-925X-10-24

[33] Kirwan, M., Duncan, M.J., Vandelanotte, C. and Mummery, W.K. (2012) Using smartphone technology to monitor physical activity in the 10,000 Steps Program: A matched case-control trial. Journal of Medical Internet Research, 14, e55. doi:10.2196/jmir.1950

[34] Luxton, D.D., McCann, R.A., Bush, N.E., Mishkind, M.C. and Reger, G.M. (2011) mHealth for mental health: Integrating smartphone technology in behavioral healthcare. Professional Psychology: Research and Practice, 42, 505512. doi:10.1037/a0024485

[35] Liu, C., Zhu, Q., Holroyd, K.A. and Seng, E.K. (2011) Status and trends of mobile-health applications for iOS devices: A developer's perspective. The Journal of Sys- 
tems and Software, 84, 2022-2033.

doi:10.1016/j.jss.2011.06.049

[36] Correa, F. (2012) Medical apps not easily defined, regulated. News and Views That Matter to Physicians. Family Practice News.

http://www.clinicalpsychiatrynews.com/views/commenta ries/single-article/the-quiet-revolution-of-mobile-health-a pplications/fbb091536048c1f718c579784baa1684.html?tx ttnews[sViewPointer] $=1$

[37] Chasky, L. and Sachs, S. (2012) Happtique releases draft app certification program standards for public comment.
Press Releases, Happtique.

http://www.happtique.com/2012/07/11/happtique-releases -draft-app-certification-program-standards-for-public-co $\underline{\text { mment }}$

[38] Carolyn, R. and Beth, B. (2011) Desired features of smartphone applications promoting physical activity. Telemedicine \& e-Health, 17, 801-803.

[39] Prochaska, J.O. and Velicer, W.F. (1997) The transtheoretical model of health behavior change. American Journal of Health Promotion, 12, 38-48.

doi:10.4278/0890-1171-12.1.38 